\title{
Nuevos y viejos dilemas y problemas éticos en época de pandemia
}

\author{
Dra. Delia M. Sánchez
}

\section{Introducción}

La pandemia producida por el agente SARSCoV-2, que lleva ya casi dos años de duración, ha conmovido a la humanidad, causando casi cinco millones de muertos y una alteración profunda en los ámbitos económico y social, además del estrés sobre todos los sistemas de salud del mundo.

Vista desde la bioética, de marzo de 2020 a la fecha se ha presentado una especie de compendio de todos los dilemas tradicionales de la ética de la salud pública. Si bien el virus es nuevo, los problemas éticos que plantea esta pandemia y los mecanismos para su control no lo son.

¿Qué queremos decir cuando hablamos de salud pública? Existen diversas definiciones. Una es la del Instituto de Medicina de los Estados Unidos: "Lo que nosotros, como sociedad, hacemos colectivamente para asegurar las condiciones para que las personas sean sanas"(1). Otra, propuesta por Darras, es la siguiente: "La salud pública transforma el cuerpo individual, como objeto de salud, en un cuerpo socializado y en un cuerpo social normalizado. Se pasa del tratamiento de la enfermedad a la planificación de salud"(2).

Es importante tener presente que, si bien el personal de salud trata individuos, el manejo de una pandemia es una genuina acción de salud pública, cuyo objeto de acción es la población, o sea, el conjunto de los habitantes de un territorio.

Las dos áreas clásicas de discusión bioética en salud pública se centran en el conflicto entre libertad (autonomía) individual versus bienestar colectivo, y la distribución justa de los recursos de salud disponibles.

En este contexto, la discusión entre autonomía y bienestar colectivo es diferente a la de la clínica, porque en la clínica no puede obligarse a nadie a seguir una indicación; en la salud pública, en cambio, la sociedad le otorga al Estado la potestad de policía sanitaria (con claras limitaciones), y este cuenta con el derecho y los medios para hacer cumplir su indicación.

La posibilidad de limitar los derechos individuales fundamentales en aras del bienestar colectivo ha preocupado tanto a bioeticistas como a políticos desde hace mucho tiempo. Por eso, se han propuesto algunos criterios básicos para justificar y limitar esta intrusión en la privacidad y la libertad de decisión. Según el autor, se proponen marcos de análisis deontológicos o consecuencialistas.

Nancy $\operatorname{Kass}^{(3)}$ presentó un modelo de análisis de políticas de salud pública analizando los siguientes puntos:

1) ¿Cuáles son las metas del programa de salud pública propuesto?

2) ¿Qué tan efectivo es el programa para alcanzar las metas indicadas?

3) ¿Cuáles son las cargas conocidas o potenciales del programa?

4) ¿Pueden minimizarse estas cargas? ¿Existen abordajes alternativos?

5) ¿Se implementa el programa en forma justa?

6) ¿Cómo pueden equilibrase las cargas y los beneficios de un programa?

Si bien existen otras numerosas propuestas, la lógica es siempre la misma: preferir la medida menos intrusiva sobre la privacidad y la libertad de los ciudadanos, que permita alcanzar el fin deseado; considerar todas las alternativas, basarse en el conocimiento existente, y distribuir las cargas (económicas y de molestias o riesgos) en forma equitativa entre los miembros de la población.

Respecto a la distribución de recursos de salud, se entiende la justicia como equidad, es decir, una distribución desigual que genere mayor igualdad. Esto, que parece sencillo, en un contexto de recursos limitados plantea la pregunta sobre cuál es la forma más justa de desarrollar criterios de priorización en el acceso a productos

Médica, magister en Salud Pública y en Bioética. Miembro del Comité de ética de Facultad de Medicina, Universidad de la República, Uruguay y del INDT. Miembro de la Comisión Nacional de Ética en Investigación 
y servicios, lo que, como veremos, ha sido problemático durante esta pandemia.

\section{Problemas éticos en la prevención de la enfermedad}

\section{Restricción de movilidad, cuarentenas}

A los efectos de disminuir la transmisión del virus, los distintos países tomaron medidas para restringir la movilidad de los habitantes, desde medidas relativamente moderadas, como las aplicadas en nuestro país, a la prohibición de salida del domicilio, salvo para compras imprescindibles o algunas actividades consideradas esenciales ("lockdown"). Si comprendemos que la restricción de movimiento es la pena que se impone judicialmente por haber cometido un delito, entonces apreciamos el grado de severidad e intrusión sobre la libertad que implican estas medidas.

Nuestro país optó, durante el 2020, por lo que las autoridades denominaron "libertad responsable", disminuyendo la movilidad y la presencialidad laboral y educativa, cerrando espectáculos públicos y fronteras, prohibiendo las reuniones, pero no la libertad de movimiento. De esta forma, las restricciones a las libertades individuales, si bien existieron, fueron menores que las aplicadas en una gran cantidad de países. Y en 2021, a pesar de ser el momento en el que realmente se disparó la pandemia en Uruguay, se comenzaron a abrir distintas actividades, con restricciones. En gran medida esto se pudo hacer porque, al mismo tiempo, comenzó el proceso de vacunación de la población. De todos modos, las decisiones que se tomaron en cada año no estuvieron exentas de discusión, por los riesgos a la salud o por los impactos económicos y sociales de cada medida. Estas discusiones ejemplifican los dilemas éticos de las medidas de salud pública, y a la vez la dificultad de tomar y mantener las decisiones en este campo.

El poder desplazarse entre países también es un derecho humano reconocido. Sin embargo, la mayor parte de los países del mundo mantuvieron sus fronteras cerradas más de un año, con contadas excepciones para la situación de refugiados, personal diplomático y de salud internacional.

Las cuarentenas son medidas extremas de restricción de la movilidad. Históricamente, se aplicaron en distintas epidemias, sin embargo, la duración de esta pandemia planteó la posibilidad de una cuarentena de meses, algo de lo que no existían antecedentes. Por otra parte, las decisiones de algunos países de cuarentenar a todos los tripulantes y pasajeros de embarcaciones en las que se presentaron casos de COVID-19, si bien quizás protegieran a la población en tierra, expusieron a un riesgo muy superior a las personas que se encontraban a bordo. Este es el caso del Diamond Princess ${ }^{(4)}$.

Frente a estas situaciones, resulta adecuado preguntarse si se siguieron los criterios propuestos por Kass: ¿se comenzó con la medida menos intrusiva?, las medidas ejecutadas ¿se basaban en conocimiento científico?, ¿se distribuyeron las cargas en forma equitativa? El caso del Diamond Princess obliga a preguntarse si es equitativo exponer a un grupo de personas a un riesgo mayor (mucho mayor, en este caso) que al resto de la población, justamente para beneficio de esa población expuesta a menor riesgo. La respuesta a esta pregunta depende de la teoría ética que se aplique: para un consecuencialista, si se hubiera obtenido el resultado deseado, la respuesta sería sí, mientras que para un afiliado a teorías deontológicas nunca se justificaría sacrificar a unos en beneficio de otros.

\section{Vacunación, vacunación obligatoria}

$\mathrm{Al}$ comienzo de la pandemia no se disponía de vacunas contra el nuevo agente SARS-CoV-2, pero algunas de estas se desarrollaron y aprobaron en un tiempo récord (un año), si bien se trataba de una aprobación de emergencia, sin haber completado todas las fases de investigación clínica usualmente requeridas para el registro de un medicamento nuevo.

Los Estados se vieron, entonces, enfrentados a dos dilemas respecto a la vacunación de sus ciudadanos: la utilización de productos con autorizaciones de emergencia (incluso la utilización de algunos productos que no contaban siquiera con ese nivel de autorización por agencias internacionales) y la voluntariedad u obligatoriedad de la vacunación.

Nuestro país no estuvo ajeno a este dilema, al que se sumaba la escasez mundial de vacunas, optándose por vacunar a la mayoría de la población con una vacuna que aún no contaba con aprobación por la Organización Mundial de la Salud (OMS), y reservando las dosis de vacunas que contaban con aprobación de emergencia (y habían demostrado mayor capacidad de desarrollar inmunidad) para grupos de mayor riesgo: los grupos más expuestos, como el personal de la salud, y los más vulnerables, como los ancianos, personas institucionalizadas o usuarios de refugios. La priorización de grupos a vacunar y la decisión de utilizar una u otra vacuna también planteó un problema ético. En el caso uruguayo, se partió de un criterio de justicia, de allí la priorización de 
los más expuestos y los más vulnerables, aunque otros proponían, por ejemplo, la vacunación de los adultos jóvenes primero, como forma de mantener en funcionamiento la economía.

Una vez que las vacunas fueron utilizadas con alta efectividad y relativamente pocos efectos secundarios en millones de personas en el mundo ${ }^{(5)}$, y habiéndose demostrado que constituyen una estrategia adecuada para frenar la pandemia y evitar muertes por la enfermedad, a lo que se suma que se cuenta con aprobación total (y no ya de emergencia) para alguna de ellas ${ }^{(6)}$, se ha planteado la pregunta sobre si la vacunación debería ser obligatoria para toda la población o para grupos específicos, como el personal de salud, aduciendo no solo la protección personal, sino el bienestar social, sea, por ejemplo, por la necesidad de retomar la educación presencial, suspendida más de un año en todo el mundo, o impedir la transmisión a pacientes vulnerables que estaban a cargo de personal de salud no vacunado. En Uruguay existe una tradición de vacunación obligatoria que no fue cuestionada socialmente hasta fines del siglo XX. A pesar de la existencia de un número pequeño pero creciente de población que adoptó una postura "antivacunas", los números muestran que, una vez disponibles, las vacunas tuvieron una alta aceptación de la población ${ }^{(7)}$. Sin embargo, en otros países, la idea de vacunación obligatoria no es aceptada y se deja librada a la decisión propia (o de los padres) la posibilidad de recibir o no las distintas vacunas disponibles. En una situación de pandemia, esta última posición pone en riesgo la salud del conjunto de la población. El informe del Comité Internacional de Bioética sobre Responsabilidad Individual ${ }^{(8)}$ asimila la actitud de quienes no teniendo contraindicaciones optan por no vacunarse, a los que en economía se conocen como free riders, los que se benefician de bienes o servicios comunes que el resto de la sociedad aporta, pero ellos no. Sin llegar a pedir que la vacunación sea obligatoria en todos los países, este informe lo considera (cuando es necesario, y con productos de eficacia y seguridad comprobadas) como un deber moral.

\section{Búsqueda y seguimiento de contactos}

Otra herramienta epidemiológica fundamental en una epidemia o pandemia es la búsqueda y seguimiento de contactos. Esta medida, de indiscutible utilidad, sobre todo cuando no existe circulación comunitaria del virus, entra en conflicto directo con el derecho de privacidad y la protección de la intimidad de los ciudadanos. Una vez más, siguiendo a Kass, se plantea si existe una forma menos intrusiva de seguimiento de los contagios, y si todas las formas de seguimiento son de igual manera respetuosas de los derechos de los ciudadanos.

Un caso paradigmático es el uso de aplicaciones, como Coronavirus UY, desarrollada y utilizada en nuestro país, en la que el informe de contactos es voluntario, versus una aplicación relativamente similar utilizada en una provincia de China, pero cuyo uso fue obligatorio, incluyendo la imposibilidad de deshabilitar el seguimiento de los movimientos de los ciudadanos, despertando temor de que se convierta en un instrumento de seguimiento personalizado ${ }^{(9)}$. En qué momento una medida de salud pública pasa a convertirse en una medida de control ciudadano es un dilema de difícil solución.

\section{Problemas en la distribución de recursos escasos}

El segundo dilema "clásico" en bioética y salud pública es, como se ha dicho antes, el de la distribución de recursos escasos.

Durante la pandemia de SARS-CoV-2 se presentaron dos ejemplos claros: el de la distribución internacional de vacunas, una vez que estuvieron disponibles, y el del acceso a camas de cuidados intensivos.

Respecto a las vacunas, antes de que existieran, los países se pusieron de acuerdo en la OMS para crear el mecanismo COVAX, cuyo objetivo era asegurar el acceso a vacunas a todas las poblaciones del mundo, independientemente de su poderío económico. Se trataba de una propuesta solidaria, en la que un recurso necesario, pero aún inexistente, se distribuiría según necesidad, y los países más ricos contribuirían a la adquisición para los más pobres ${ }^{(10)}$. Sin embargo, como es bien sabido, una vez que aparecieron vacunas, aún en etapas de investigación, los países con mayor poder económico se apresuraron a comprar lo que precisaba su población y más, mientras que los restantes países se encontraron sin acceso a vacunas. De allí los datos sobre cobertura que muestran que mientras algunos países (incluido el nuestro) alcanzaron más de $70 \%$ de cobertura con dos dosis de vacunas, los más pobres presentaban tasas menores al $10 \%{ }^{(7)}$. En este momento, en el que se plantea la necesidad de una tercera dosis, otra vez se relega la vacunación en los países más pobres. Sabemos que hasta que no haya protección de toda la población mundial, la pandemia seguirá su curso, por la existencia de enormes bolsones de individuos susceptibles, por lo cual, aun por motivos egoístas, es necesaria la solidaridad, pero: ¿aceptará una población ser postergada 
en beneficio de otra, quizás lejana, aunque más necesitada? En la distribución de vacunas participan otros componentes no menores: las patentes, que protegen a quienes desarrollan nuevas ideas, y que pueden convertirse en barreras infranqueables para el acceso a productos imprescindibles para la vida, cuando van unidas a mecanismos de fijación de precios que podríamos catalogar de extorsivos. Se ha planteado la eliminación de las patentes en casos de necesidad de salud pública, o, al menos, el uso de licencias compulsivas para su fabricación. Sin embargo, para que funcionen en una emergencia, es necesario que exista capacidad instalada, y no ha sido el caso. De hecho, India, uno de los pocos países del mundo con una industria de medicamentos genéricos importante, que era un elemento principal de la estrategia COVAX, se vio desbordada cuando la pandemia afectó a su población, sin poder dar cuenta de la producción originariamente prevista ni tampoco exportar las cantidades suficientes por la demanda interna.

El otro ejemplo de dilemas en la distribución de recursos escasos surgió ante el crecimiento de la demanda de camas de cuidados intensivos. Algunos países, como el nuestro, tuvieron tiempo de aumentar su capacidad instalada antes del momento de mayor demanda (si bien con dificultades para la cobertura de personal). No obstante, otros, a quienes la pandemia alcanzó primero, vieron sus servicios de salud desbordados y muy especialmente sus servicios de tratamiento intensivo. Y entonces aparecieron guías para la selección de pacientes. El dilema ético no es la existencia de guías; en la práctica clínica diaria suelen utilizarse algoritmos de toma de decisiones para determinar qué pacientes se beneficiarán de un tratamiento y por lo tanto se les ofrecerá, y en quiénes sería un gesto fútil y, por ende, desaconsejable. Pero en el límite, cuando se planteaba una sola cama para dos personas con igual necesidad y posibilidades de beneficio, entró en escena el concepto de diferente valor social, o sea, el de diferente valor de la vida de los seres humanos. No deberíamos hablar aquí de un dilema, porque un dilema no implica elegir entre lo malo y lo bueno, sino entre dos cosas buenas e imperfectas. Lo que se "coló" fue una idea economicista de la vida humana, en la que se valora su potencial contribución a la sociedad como fundamento de su dignidad (y, por lo tanto, un mayor derecho a que se proteja su vida), en contraposición al concepto básico kantiano y de derechos humanos de la igual dignidad (por lo tanto, igual valor y derechos) de todos los seres humanos sin excepción. El ejemplo más cla- ro de esto se encuentra en la guía de la Sociedad Española de Medicina Intensiva, Crítica y de Unidades Coronarias $^{(11)}$. El punto 23 de las recomendaciones generales de selección de pacientes de dicha guía dice: "Se debe tomar en cuenta el valor social de la persona enferma". No se define cuál es ese "valor social", ni quién es el encargado de medirlo, sin embargo, se sobreentiende: el marginal, el anciano, el adicto, el pobre, el desempleado, el que está solo, deben tener menor "valor social" porque no contribuyen al bienestar general y, entonces, no los precisaría nadie. Por si eso fuera poco, la misma guía también presenta criterios de "edadismo" y "ableismo", proponiendo priorizar a quienes tengan una mayor esperanza de vida (indicador válido para poblaciones, pero no para individuos) y mayor probabilidad de vida libre de discapacidad. Discutí los problemas éticos de esta visión utilitarista de la vida humana en un podcast de la Redbioética sobre Ética y Pandemia ${ }^{(12)}$.

\section{Problemas de ética de la ciencia}

Si bien no se trata de los problemas éticos más habitualmente reconocidos en un contexto de pandemia, existen dudas sobre cómo actuar que se enmarcan en lo que conocemos como Ética de la ciencia.

En cuanto a la investigación en contexto de pandemias, los dilemas se plantean en el momento de diseñar estudios de nuevos productos: ¿se debe investigar en las poblaciones más afectadas (en mayor situación de vulneración de salud) o en las menos afectadas? ¿En los individuos más vulnerables biológicamente o en los más fuertes? ¿Cómo invitar a participar en una investigación sin generar falsas expectativas? ¿Es ético acelerar el proceso de aprobación de una investigación o, por el contrario, arriesgar a una población a sufrir los efectos de una epidemia durante más tiempo? La OMS publicó guías éticas para la investigación en contexto de epidemias, que es interesante conocer $^{(13)}$. Un punto fundamental es distinguir con claridad el rol y las potestades de la autoridad sanitaria de los de otros investigadores, ya que a la autoridad sanitaria se le permiten excepciones que no son válidas para otros.

En la epidemia de COVID-19, y con el propósito de acortar los tiempos de investigación de vacunas, se propusieron (y en algunos casos se realizaron) investigaciones de "desafío". Este modelo de investigación se basa en inocular a voluntarios sanos la vacuna que se está estudiando y luego exponerlos al agente infeccioso. No es el modo habitual de investigar vacunas, pero sus propo- 
nentes, utilizando un criterio utilitarista, según el cual el sacrificio de unos pocos se justificaría por el bien de muchos, aducían que, tratándose de personas jóvenes y sanas, el riesgo era relativamente pequeño. Sabemos, sin embargo, que hasta el momento actual no existe un tratamiento comprobado para la enfermedad, por lo que, en caso de enfermar, se exponía incluso a la muerte a los voluntarios. Solbakk y cols. exploraron en detalle los argumentos éticos contrarios a esta práctica ${ }^{(14)}$.

Otro dilema de ética científica durante la pandemia fue el crecimiento de artículos científicos publicados en repositorios sin ser sometidos a revisión por pares. Justificado por la enorme producción científica en torno al SARS-CoV-2, que imposibilitaba que todo lo redactado se revisara de manera apropiada y se publicara en forma oportuna, se recurrió como nunca antes a la publicación sin revisión, en repositorios. Esto dio origen a que muchas investigaciones con problemas metodológicos, que no hubieran sido usualmente aceptadas para su publicación, estuvieran disponibles y en términos de igualdad con estudios bien realizados. Es difícil saber con certeza cuál es el nivel de equilibrio ideal entre la rápida disponibilidad de resultados de investigación versus la circulación de información científica falsa (o al menos de dudosa rigurosidad) en una situación de emergencia.

La baja exigencia en la información circulante también dio pie a la utilización de tratamientos no comprobados, surgidos de estudios realizados sin cumplir con todos los requisitos de una investigación clínica de calidad. Son conocidos los casos de la hidroxicloroquina o la ivermectina, entre otros. En estos días se ha difundido en los medios una denuncia del Consejo Nacional de Salud y la Comisión Nacional de Ética en Investigación con Seres Humanos de Brasil, relativa a la realización sin autorización de estudios locales de hidroxicloroquina, incluso sin que los pacientes supieran que estaban participando en investigación y presionando a los médicos para que la indicaran ${ }^{(15)}$.

\section{Comentarios finales}

Como hemos podido ver, la pandemia de COVID-19 ha planteado todos los problemas de la ética de la salud pública, además de problemas de ética clínica y científica.

El dilema siempre existente entre los derechos individuales y el bienestar colectivo es de muy difícil solución, aunque no por ello imposible de resolver, si pensamos en los seres humanos como seres en comunidad, responsables unos por otros, pero al mismo tiempo como depositarios de una dignidad intrínseca, sin grados de valor entre unos y otros.

En tiempos de pandemia, cuando las sociedades y cada individuo dentro de ellas se sienten más amenazados y vulnerables, no es tiempo de renunciar a los valores y principios éticos, sino de ponerlos en práctica. Aunque la tentación de "saltearse pasos" que se nos presenta parezca muy fuerte, hemos aprendido que puede llevarnos a situaciones en extremo injustas, peligrosas y pobres desde el punto de vista científico.

\section{Delia Sánchez, ORCID 0000-0002-4094-8344}

Este artículo fue aceptado para su publicación por el Editor jefe, Dr. Federico Ferrando

\section{Bibliografía}

1. Institute of Medicine. The Future of Public Health. Washington, D.C.: National Academy Press; 1988.

2. Darras C. Bioética y Salud Pública. Al cruce de los caminos. Acta Bioeth. 2004; 10 (2): 227-33. doi: 10.4067/S1726-569X2004000200010.

3. Kass NE. An ethics framework for public health. Am J Public Health. 2001; 91 (11): 1776-82. doi: 10.2105/ajph.91.11.1776.

4. Nakazawa E, Ino H, Akabayashi A. Chronology of COVID-19 cases on the Diamond Princess cruise ship and ethical considerations: a report from Japan. Disaster Med Public Health Prep. 2020;14 (4): 506-13. doi: 10.1017/dmp.2020.50.

5. Joi P. How safe are COVID vaccines? GAVI. The vaccine Alliance [internet]. 2021 [consultado el 11 de octubre de 2021]. Disponible en: https://www. gavi.org/vaccineswork/how-safe-are-covid-19-vaccines.

6. U.S. Food and Drug Administration. FDA approves first COVID19 vaccine [internet]. 2021 [consultado el 11 de octubre de 2021]. Disponible en: https://www.fda.gov/news-events/press-announcements/fda-approves-first-covid-19-vaccine.

7. OMS. WHO Coronavirus (COVID-19) dashboard [internet]. 2021 [consultado el 11 de octubre de 2021]. Disponible en: https://covid19.who.int/.

8. Comité Internacional de Bioética de UNESCO. Report of the IBC on the Principle of Individual Responsibility as related to Health. UNESCO: París; 2019 [consultado el 11 de octubre de 2021]. Disponible en: https://unesdoc.unesco.org/ark:/48223/ pf0000367824 Acceso el 11/10/2021.

9. Zhou SL, Jia X, Skinner SP, Yang W, Claude I. Lessons on mobile apps for COVID-19 from China. Journal of Safety Science and Resilience. 2021; 2 (2): 409. doi: 10.1016/j.jnlssr.2021.04.002.

10. OMS. COVAX: colaboración para un acceso equitativo mundial para las vacunas contra la COVID19 
[internet]. 2020 [consultado el 7 de octubre de 2021]. Disponible en: https://www.who.int/es/initiatives/act-accelerator/covax.

11. Rubio-Sanchíz O, Estella-García A, Martín-Delgado MC, Saralegui-Reta I, Cabré-Pericas L, Zapata-Fenor L. Recomendaciones éticas para la toma de decisiones en la situación excepcional de crisis por pandemia COVID-19 en las unidades de cuidados intensivos [internet]. SEMICYUC. 2020 [consultado el 5 de octubre de 2021]. Disponible en línea en: https://semicyuc.org/wpcontent/ uploads/2020/03/\%C3\%89tica_SEMICYUC-COVID-19.pdf.

12. Sánchez D. Ética y pandemia. Podcast de la Red bioética UNESCO [internet]. T1 C4. 16 de abril de 2020 [consultado el 10 de octubre de 2021]. Disponible en: https://redbioetica.com.ar/podcast-redbioetica-temporada-1-etica-y-pandemia/.

13. OMS. Ethics in epidemics, emergencies and disasters: research, surveillance and patient care. Training manual [internet]. WHO: Ginebra; 2015 [consultado el 10 de octubre de 2021]. Disponible en: https://www. who.int/publications/i/item/ethics-in-epidemics-emergencies-and-disasters-research-surveillance-and-patient-care-training-manual.

14. Solbakk JH, Bentzen HB, Holm S, Heggestad AKT, Hofmann B, Robertsen A et al. Back to WHAT? The role of research ethics in pandemic times. Med Health Care Philos. 2021; 24, 320. doi: 10.1007/s11019-020-09984-x.

15. CONEP. Ofício No 829/2021/CONEP/SECNS/MS. Indícios de irregularidades na condução do estudo protocolo CAAE 41909121.0.0000.5553, sob título "The proxa-Recue AndroCoV Trial" [internet]. Ministerio da Saúde: Brasília, 03 de setiembre de 2021 [consultado el 10 de octubre de 2021]. Disponible en: https://sei. saude.gov.br/sei/controlador_externo.php?acao=documento_conferir\&codigo_verificador $=0022580072 \&$ codigo_crc $=18158 \mathrm{CBC} \&$ hash_download $=97 \mathrm{ec}$ $9380511 \mathrm{f} 1 \mathrm{a} 1922$ ea $2 \mathrm{f} 639 \mathrm{~d} 3667261$ a ad $900 \mathrm{~d}$ 28626f6693321eb7d51d82b0aa33d8ac66d2747bf75add37faa161f8ec728c634a6423888fd937142451a20f\&visualizacao=1\&id_orgao_acesso_externo $=0$.

La autora elaboró en su totalidad el presente artículo. 